\title{
Alternative Mechanisms for Solvation Dynamics of Laser-Induced Electrons in Methanol
}

\author{
L. Turi, P. Holpár, and E. Keszei* \\ Department of Physical Chemistry, Eötvös University, P.O. Box 32, H-1518, Budapest 112, Hungary
}

Received: December 18, 1996; In Final Form: May 19, $1997^{\otimes}$

\begin{abstract}
A multiphoton ionization study of neat methanol with subpicosecond 2-eV laser pulses has been previously reported. A hybrid electron solvation mechanism combining both a stepwise transition between two electronsolvent configuration states and a continuous first-order blue shift of the electron absorption spectra was found to closely fit the experimental data. If substantial absorption from free electrons is assumed in this spectral region, we find that another model comprising thermalization prior to a stepwise branching localization without blue-shifting spectra fits equally well at all wavelengths. However, these two models display considerable differences between their respective kinetic parameters, especially the electron localization time. Furthermore, for the nonshifting model, this calculated localization time is considerably longer than that for electron hydration in neat water. We suggest independent studies such as ultrafast electron scavenging experiments before adopting a particular mechanism for electron solvation in methanol.
\end{abstract}

\section{Introduction}

Dynamic events during electron solvation were interpreted in many different ways. The main source of variety of interpretation was the lack of an appropriate time resolution in earlier experiments. In a recent paper, ${ }^{1}$ Pépin $e t$ al. reported a thorough subpicosecond pump-and-probe multiphoton ionization experiment of pure methanol. With this first time-resolved experiment on electron solvation in neat methanol at room temperature, they have obtained extensive time-dependent transient absorption data from 400 to $1350 \mathrm{~nm}$, an exceptionally wide wavelength range. Trying to interpret the experimental data, they found that the measured kinetic curves did not support a previously suggested simple two-step mechanism of solvation but that these data could accurately be reproduced with a "hybrid" solvation model characterized by two localized species. Both species have continuously blue-shifting spectra, but during this spectral relaxation, the "weakly bound" species undergoes a stepwise transition to the "strongly bound" species, which, at the end of the spectral relaxation, becomes the fully solvated electron. Another feature of this model is a branching between a "direct" solvation path to the "strongly bound" species and an "indirect" one via the "weakly bound" species. In this paper we try to find out if the observed kinetic traces necessarily imply localized electronic species with blue-shifting spectra.

The debate whether electron solvation proceeds via different localized species, each of them having a distinct spectrum, or if there is only one localized species whose spectrum is undergoing a continuous blue shift dates back to pioneering pulse-radiolysis studies of electron solvation. ${ }^{2-9}$ Many of these early studies suggested that a continuous blue shift of the localized electron is responsible for the spectral changes observed, ${ }^{6-8}$ but some authors preferred a stepwise transition from a "presolvated" localized state to a final solvated electron. ${ }^{2-5}$ The necessity to include both the stepwise procedure and the continuous blue shift to successfully interpret the experiments was also suggested based on results obtained in cold glassy alcohols. ${ }^{9}$ The advent of the subpicosecond pump-and-probe laser technique enabled obtaining transient spectra in liquids at room temperature and most importantly in pure water, the fastest known solvent. ${ }^{10-15}$ Surprisingly, a simple two-step model

${ }^{\otimes}$ Abstract published in Advance ACS Abstracts, July 1, 1997. quantitatively explained these experimental findings. This model was usually written as

$$
\mathrm{e}_{\text {free }}^{-} \stackrel{T_{1}}{\longrightarrow} \mathrm{e}_{*}^{-} \stackrel{T_{2}}{\longrightarrow} \mathrm{e}_{\mathrm{s}}^{-}
$$

Here, $\mathrm{e}_{\text {free }}^{-}$means the delocalized "free" or "dry" electron, $\mathrm{e}_{*}^{-}$is a localized species called "wet" or "presolvated" electron, and $\mathrm{e}_{\mathrm{s}}^{-}$is the equilibrium solvated electron. In the quantitative evaluation of the ultrafast laser results, $\mathrm{e}_{\text {free }}^{-}$was a nonabsorbing species in the studied wavelength region, while to $\mathrm{e}_{*}^{-}$ and $\mathrm{e}_{\mathrm{s}}^{-}$, "static" (i.e., non-blue-shifting) absorption spectra were assigned. However, later studies with better time resolution and accuracy revealed that this simple mechanism was not sufficient to quantitatively describe some features of electron hydration. An alternative more complex mechanism including several electronic species which satisfactorily described the new experimental findings was then considered. ${ }^{16,17}$

Meanwhile, Mataga and co-workers ${ }^{18,19}$ studied the photoionization of aromatic amines in alcohols. They also found that mechanism 1 was insufficient to fully describe their results with respect to electron solvation in alcohols. They proposed the incorporation of an additional species and brought back the idea of the continuous blue shift within the following mechanism:

$$
\mathrm{e}_{\mathrm{qf}}^{-} \rightarrow \mathrm{e}_{\mathrm{loc}}^{-} \stackrel{T_{\mathrm{D}_{2}}}{\longrightarrow} \mathrm{e}_{\mathrm{ps}}^{-} \frac{T_{\mathrm{L}_{1}}}{\text { continuous shift }} \mathrm{e}_{\mathrm{s}}^{-}
$$

Here, $\mathrm{e}_{\mathrm{qf}}^{-}$is the "quasi-free" electron and $\mathrm{e}_{\mathrm{loc}}^{-}$is localized in a shallow trap, and it becomes "partially solvated" $\left(\mathrm{e}_{\mathrm{ps}}^{-}\right)$by a rearrangement of nearby solvent molecules characterized by the rotational dielectric relaxation time $T_{\mathrm{D}_{2}}$. Finally, the partially solvated species becomes fully solvated $\left(\mathrm{e}_{\mathrm{s}}^{-}\right)$in an additional rearrangement of the solvent molecules characterized by the longitudinal dielectric relaxation time $T_{\mathrm{L}_{1}}$, but this latter process is accompanied by a continuous spectral shift. A quite similar model successfully described the three-pulse experiments of Barbara and co-workers ${ }^{20}$ when they studied the relaxation of excited solvated electrons in methanol. In their interpretation, the excited (p-state) electron first tranfers to a "partially desolvated s-state", then the ground-state relaxes, restoring back the fully equilibrated solvated state. This ground-state solvation is modeled by a continuous spectral shift. 


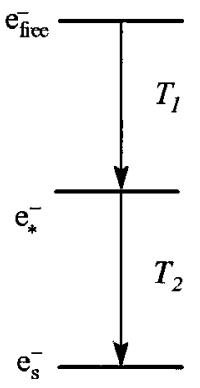

(1)

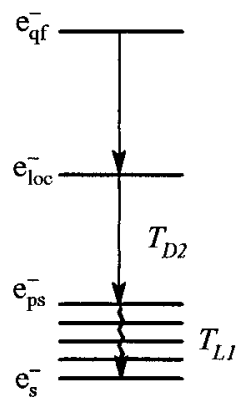

(2)

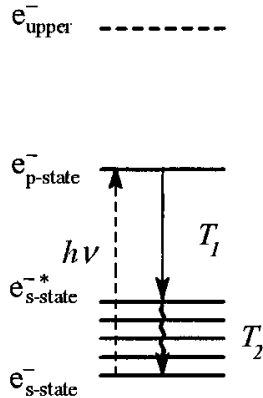

(2')

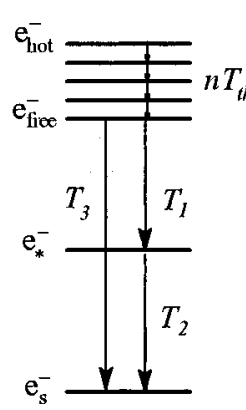

(3)

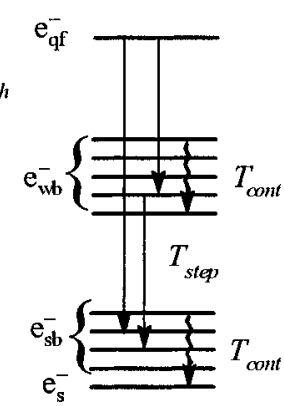

(4)

Figure 1. Schematic representation with energy level diagrams of five different electron solvation mechanisms discussed, identified with the same number as used in the text: (1) two-step mechanism, e.g., Shi et al., ${ }^{29}$ (2) three-step mechanism, Hirata et al., ${ }^{18,19}\left(2^{\prime}\right)$ direct excitation of $\mathrm{e}_{\mathrm{s}}^{-}$, Walhout et al.,$^{20}$ (3) thermalization and branching, present paper, (4) "hybrid" branching with continuous shift and stepwise transition, Pépin et al. ${ }^{1}$ Straight arrows indicate stepwise transitions, while waved arrows represent continuous spectral shifts. Identificaton of the species can be found in the Introduction after the corresponding scheme.

Besides the laser experiments, extensive molecular dynamics simulation studies of electron solvation have also been performed. ${ }^{21-26}$ Most of the studies modeled electron solvation in water, and a reasonable agreement of the simulation results with ultrafast laser experiments was obtained from diabatic quantum-dynamic simulations. ${ }^{24-26}$ Two major aspects have been assumed in the diabatic simulation results of Rossky and co-workers. ${ }^{26,27}$ First, that there are two channels of localization, one to an excited localized state and another to the localized ground state. Second, that there is a nonzero contribution to the absorption in the near-infrared-visible range of the delocalized manifold, in addition to the localized species. This mechanism can be written as follows:

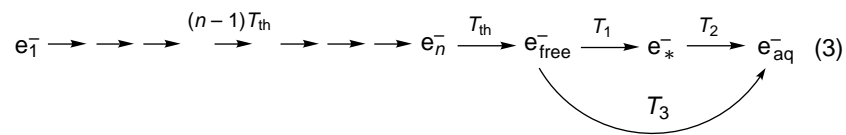

Here, $\mathrm{e}_{1}^{-}$to $\mathrm{e}_{n}^{-}$are "hot" electrons with excess kinetic energy, $\mathrm{e}_{1}^{-}$getting thermalized in $n-1$ steps to become $\mathrm{e}_{\text {free }}^{-}$. The $\mathrm{e}_{\text {free }}^{-}$species has lost enough energy to get localized either in the $\mathrm{e}_{*}^{-}$"localized excited state" or in the $\mathrm{e}_{\mathrm{s}}^{-}$"localized ground state". Analysis of the simulated electron trajectories also showed that the final localization steps are mainly stepwise, and "spectral changes which occur after the nonadiabatic transition are of little importance, ... for most purposes the spectrum of the hydrated electron after relaxation (of the electron) to the ground state is, in fact, the equilibrium spectrum". ${ }^{26}$ Mechanism 3 very successfully described experimental ultrafast laser results on the solvation of electrons in pure water and led to reasonable kinetic parameters with the added benefit of deducing for the first time the absorption spectra of the "hot" and free electrons in the near-infrared and visible regions. ${ }^{28}$

Due to the limited wavelength coverage, low yield of aqueous electrons, and most importantly the ultrafast nature of the reaction, it becomes very difficult to discriminate between suitable models. In particular no attempt was made to apply a model which includes a spectral shift in spite of serious indications from low-temperature studies in alcohols. Since electron hydration is a process taking place on a time scale comparable to the laser pulse width, a study in a polar liquid where solvation is an order of magnitude slower would constitute a careful approach in the effort to properly describe electron solvation. Pépin et al. ${ }^{1}$ have chosen pure methanol to study electron solvation, where the temporal resolution seemed to be sufficient to satisfactorily measure most details of the early events of solvation. Using different detection techniques, they also measured kinetic curves in an unusually wide wavelength range from 400 to $1350 \mathrm{~nm}$. When trying to interpret the observed data on the basis of the two-step mechanism (1), they found that the parameters $T_{1}$ and $T_{2}$ thus obtained varied monotonically from one wavelength to the other, with about a 4-fold variation between the limits of the covered wavelength range. This means that a global fit for all wavelengths of mechanism 1 is impossible. They also observed a marked blue shift of the transient spectra, which has led to a model similar to the one once suggested by Ogasawara et al., ${ }^{9}$ a branching combined with continuous blue shift of the localized species' spectra, with a concomitant stepwise transition that can be depicted as follows:

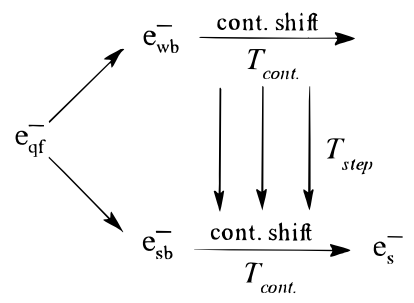

Here, $\mathrm{e}_{\mathrm{qf}}^{-}$is the quasi-free electron which localizes into one of the "weakly bound" $\left(\mathrm{e}_{\mathrm{wb}}^{-}\right)$and "strongly bound" $\left(\mathrm{e}_{\mathrm{sb}}^{-}\right)$states. Both species are subject to solvent relaxation, resulting in a continuous blue shift of their spectra. Meanwhile, $\mathrm{e}_{\mathrm{wb}}^{-}$is transformed into $\mathrm{e}_{\mathrm{sb}}^{-}$in a stepwise transition. This model perfectly described all experimental observations in the entire wavelength range without having to include transient absorption spectra for the "hot" and free electrons like in all the other models except mechanism 3.

In order to illustrate differences between electron solvation mechanisms, we show their energy level diagram in Figure 1. The simplicity of mechanism 1 is clearly seen, compared to the others, which are more complex and hence more flexible to fit to the experimental data. Models 2 and 4 include a continuous spectral shift in the solvation. In scheme 2 the shift only occurs at the end of the solvation process, but in scheme 4 relaxation simultaneously affects the spectra of both localized species. Although Figure 1 might suggest that these two electronic species are actually two levels lying in the same potential well, Pépin et al. consider that they could also represent two distinct configuration states with different binding energies $\left(\mathrm{e}_{\mathrm{wb}}^{-}\right.$and $\left.\mathrm{e}_{\mathrm{sb}}^{-}\right)$between which a stepwise conversion occurs from the weakly bound to the strongly bound type. 
The thermalization of mechanism 3 on the manifold of excited states would involve a pseudocontinuous shift which was modeled by $n$ discrete thermalization steps with uniform characteristic times $T_{\text {th }}$ and the absorptivities of all the species in the thermalization manifold being kept identical. The main formal difference between models 3 and 4 thus lies in the location of the continuous (or quasicontinuous) shift in the energy of the electronic species. One conceptual difference between mechanisms 3 and 4 is that model 3 only includes a quasicontinuous shift in the form of the discrete thermalization manifold, all (localized and nonlocalized) species' spectra being essentially time-independent. In fact, model 3 attributes the apparent blue shift of the measured transient spectra at early times $(10-20 \mathrm{ps})$ mainly to a transition from $\mathrm{e}_{\text {free }}^{-}$to $\mathrm{e}_{*}^{-}$, and a smaller portion to the transition from $\mathrm{e}_{*}^{-}$to $\mathrm{e}_{\mathrm{s}}^{-}$. Since the "hot" species contributes to the absorption only within the first picosecond, the quasicontinuous shift (thermalization manifold) does not contribute to the apparent blue shift of the observed transient spectra to an important degree.

The question arises as to what extent the interpreting power of mechanism 4 can be considered as a justification for the blueshifting mechanism. A renewed interest over this issue has emerged since the recent publication of ultrafast laser results on electron solvation in pure methanol, in which Shi et al. ${ }^{29}$ basically support the simple two-step mechanism (1), with an additional "ground state cooling" after the second step. However, this final ground-state cooling is very slow, having a characteristic time of $40 \mathrm{ps}$, compared to $7.1 \mathrm{ps}$ for solvation.

The aim of this paper is to fit the kinetic curves of electron solvation in neat methanol reported by Pépin et al., ${ }^{1}$ to see if they are in accordance with mechanism 3 and furthermore if they necessarily imply blue-shifting spectra of the localized electronic species. First we briefly describe the analyzed experimental data and the applied numerical methods in detail. After the subsequent presentation of the results, we discuss the applicability of different solvation mechanisms.

\section{Experimental and Numerical Details}

Technical details concerning the experiments of Pépin et al. can be found elsewhere. ${ }^{1}$ Here we recall that they performed multiphoton ionization of pure methanol at $294 \mathrm{~K}$, with 625$\mathrm{nm}(2-\mathrm{eV})$ laser pulses of approximately $300 \mathrm{fs}$ fwhm, with an irradiance of $\sim 10^{13} \mathrm{~W} / \mathrm{cm}^{2}$ inside the sample cell. Within these conditions, at least three photons are needed to reach the 4.7$\mathrm{eV}$ threshold of ionization. This low-energy pulse had some important implications. There were some specific wavelengths where Raman emission or absorption occurred. These Raman peaks appeared strictly at synchronized pump-and-probe pulses, so they assured an exact determination of zero time delay. On the other hand, obviously due to the possibility of the formation of relatively low excess kinetic energy electrons, there was evidence of geminate recombination of electrons. This has been taken into account with a survival probability of a linear combination of three exponentials found when comparing Monte Carlo simulations of the recombination with the experimental data. ${ }^{1}$ For the sake of clarity, we have removed the Raman peaks from the experimental data points before performing the kinetic analysis. The resulting kinetic curves are shown in a three-dimensional diagram in Figure 2. These curves are still distorted by the convolution of the "instantaneous" kinetic curves with the instrumental response function, which is an "effective pulse" in our case. The consequences of this convolution are discussed in details in ref 28 . The width of the effective pulse was determined from the Raman peaks.

The only possibility to perform the necessary deconvolution to get the instantaneous kinetic curves is the method usually
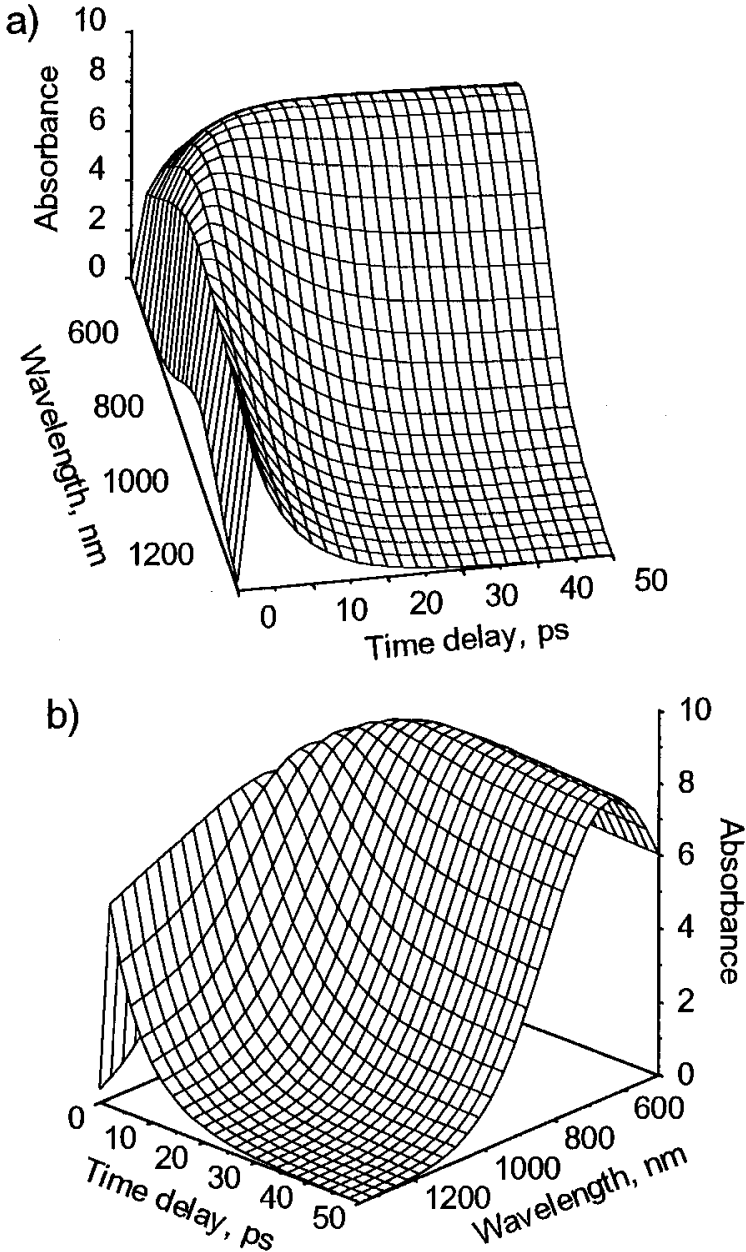

Figure 2. Three-dimensional representation of the measured pumpand-probe absorbancies for the solvation of the electron following ionization in neat methanol at $294 \mathrm{~K}$ : (a) mainly shows a view of the kinetic traces (time dependence) while (b) mainly shows the transient spectra (wavelength dependence). The surface is constructed after removal of the Raman peaks and a smoothing of the experimental noise (which can be seen in Figure 3), to show the overall variation of the $\triangle \mathrm{OD}$ signals as a function of time delay between the probe and the pump pulses, and wavelength. Note the apparent blue shift of the absorbance maximum with increasing time delay. Absorbance units are normalized to $1 \mathrm{~mol} / \mathrm{dm}^{3}$ electrons in $1000 \mathrm{M}^{-1} \mathrm{~cm}^{-1}$ units.

called reconvolution, ${ }^{28}$ or maximum likelihood deconvolution. ${ }^{30}$ This means that one should provide an a priori kinetic model with its set of kinetic and optical data as parameters and then convolute the obtained ("calculated") instantaneous curves with the effective pulse. The difference between the calculated convolution and the experimental curves is then minimized by optimizing the kinetic and optical parameters. Using this technique, deconvolution and parameter estimation are performed in one step, but one cannot make a deconvolution without supposing a particular mechanism. Keeping this in mind, we can decide the applicability of one mechanism or another only by finding out if it can be satisfactorily fitted to the convolved experimental data. This is the way we followed when testing the applicability of a particular mechanism. To see if the hypothesis of continuously blue-shifting localized electronic species spectra is necessary to explain the experimental data, we tried to fit mechanism 3 , which does not contain these blue-shifting spectra.

When performing the fit of a complex mechanism (like (3) or (4)) to experimental data, we usually have an ill-conditioned parameter estimation problem. This means that experimental data could be described with fewer parameters than we actually 
put in, so a certain number of parameters will be undetermined (or not well-determined). To circumvent this problem, we have to impose some constraints on the kinetic and/or optical parameters. To this end, the optimization of the parameters of mechanism 3 has been performed in two steps. First, the estimation of parameters was done at each single wavelength with a suitable fixed set of kinetic parameters optimizing three unknown molar absorptivities (that of $\mathrm{e}_{\text {hot }}^{-}, \mathrm{e}_{\text {free }}^{-}$, and $\mathrm{e}_{*}^{-}$) and the fine position of the zero delay time of the effective pulse. For the kinetic curves at 13 different wavelengths, this resulted in 13 molar absorptivities for each of the three species. We then readjusted the shape of optical spectra to obtain reasonably smooth curves. This spectral shape was then fixed in the second step of optimization, and the estimation was performed simultaneously for all 13 wavelengths. Parameters to optimize were then the four kinetic parameters $\left(T_{\mathrm{th}}, T_{1}, T_{2}, T_{3}\right)$, and a factor for each spectrum to scale the absorptivities with the fixed spectral shape, for a total of seven parameters. Thus, spectral shapes did not change in this second step, only their amplitude. The spectrum of the solvated species was taken from the literature. ${ }^{31}$ Steps 1 and 2 were repeated until a satisfactory fit was found for which $T_{1}<T_{2}$ (this condition leads to reasonable oscillatory strengths). All in all, the ill-conditioned estimation was avoided by forcing a smooth spectral shape for each transient absorbing species.

We would like to point out some particularities of model 3 . Most important is to emphasize that we attributed a nonzero absorbance to all of the electronic species involved, unlike the usual treatment of forcing zero absorbance on electrons prior to localization. So we assigned a distinct absorbance spectrum to $\mathrm{e}_{\text {hot }}^{-}$and $\mathrm{e}_{\text {free }}^{-}$, in addition to the two localized species $\mathrm{e}_{*}^{-}$ and $\mathrm{e}_{\mathrm{s}}^{-}$. Quantum-dynamical simulation results support this idea. ${ }^{24,27-28}$ Another characteristic feature of the mechanism is that thermalization of the electron is treated as a series of finite energy deposition steps. As a consequence, the number of these steps, $n$, is a discrete variable. As the applied Marquardt algorithm for parameter estimation is only capable of treating continuous variables, the number of individual thermalization steps must be fixed during parameter estimation. In an earlier study of the simulation results of electron solvation in water ${ }^{27}$ it has been found that the fit is not very sensitive for the value of $n$, if it is higher than 10 . Nevertheless, the actual value of $n$ does not change the overall thermalization time, $n T_{\text {th }}$. In fact, it is $n T_{\text {th }}$ which has a physical meaning rather than the individual $T_{\text {th }}$ values. Note, however, that $n T_{\text {th }}$ cannot be interpreted any more as the characteristic time necessary to decrease the concentration of the appropriate species to its $1 / \mathrm{e}$ portion. The actual proportion is somewhat more than 1/e, slightly depending on the value of $n^{27}$ ( 0.458 for $n=10,0.470$ for 20 , and 0.479 for 40). The photophysical consequence of the arbitrary choice of $n$ was that we assigned one single absorption spectrum to all thermalizing species $\mathrm{e}_{1}^{-}$to $\mathrm{e}_{n}^{-}$, which we kept constant throughout the thermalization. As mechanism 3 includes two channels of localization, there is no single "localization time" only an "overall" localization time can be given as the harmonic sum of $T_{1}$ and $T_{3}$. The same branching is also included in the "blueshifting" mechanism (4). Accordingly, we will consider $n T_{\text {th }}$ as the "overall thermalization time" and $T_{\mathrm{loc}}$ as the "overall localization time".

\section{Results and Discussion}

First we fitted the simple two-step mechanism to the experimental data. Even if we allowed the pulse width to vary as a fitting parameter, we confirmed the findings of Pépin et $a l .:^{1}$ although the simple two-step mechanism fits well at

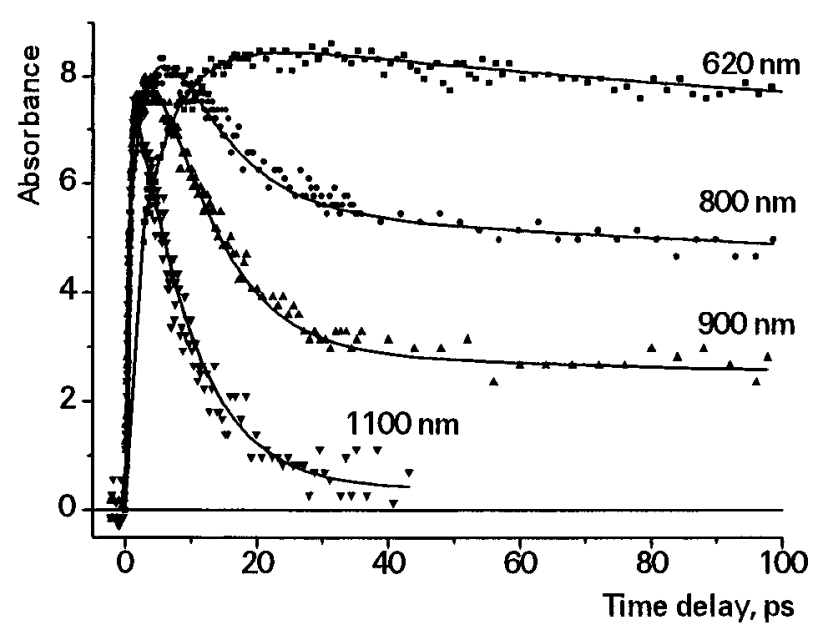

Figure 3. Fit of mechanism 3 to the experimental data points at four selected wavelengths out of the 13 that have been analyzed. The quality of the fit was similar at the other wavelengths. For the explanation of the scales see Figure 2.

TABLE 1: Kinetic Parameters of Mechanism 3 for the Solvation of Electron in Neat Methanol ${ }^{a}$

\begin{tabular}{cccc}
\hline & \multicolumn{2}{c}{ methanol } & \\
\cline { 2 - 3 } parameter & present paper & ref 1 & water ref 28 \\
\hline$n T_{\text {th }}$ & $0.56(0.01)$ & & 0.08 \\
$T_{\text {loc }}$ & $3.9(0.16)$ & $T_{\text {loc }}<1$ & 0.08 \\
$T_{1}$ & $5.9(0.31)$ & & 0.10 \\
$T_{2}$ & $8.4(0.40)$ & $T_{\text {step }}=6.1$ & 0.33 \\
$T_{3}$ & $11.7(0.70)$ & $T_{\text {cont }}=13.6$ & 0.35 \\
$\tau_{\mathrm{s}}$ & 10.3 & & 0.457
\end{tabular}

${ }^{a}$ Numbers in parentheses indicate $95 \%$ confidence intervals. The overall thermalization time ( $\mathrm{e}_{1}^{-}$to $\left.\mathrm{e}_{\text {free }}^{-}\right)$is $n T_{\text {th. }}$. The overall localization time $T_{\mathrm{loc}}$ is the harmonic sum of $T_{1}$ and $T_{3}$, i.e., $1 /\left(1 / T_{1}+1 / T_{3}\right)$. The solvation time $\tau_{\mathrm{s}}$ is the time when only 1/e part of the electrons remains unsolvated. For comparison, corresponding values of the continuous blue shift mechanism $4^{1}$ and characteristic times of mechanism 3 in pure water ${ }^{28}$ are also given. All characteristic times are given in ps units.

individual wavelengths, very different wavelength dependent characteristic times $T_{1}$ and $T_{2}$ result. This means that the twostep mechanism is a good model only in a relatively small wavelength range, similar to a relatively good local linear evaluation of a nonlinear function.

With our data, we have found that the spectral relaxation of the final species is not sufficient to quantitatively interpret the kinetic traces in the entire wavelength range, so we ruled out mechanism 2.

Next we performed the fit of mechanism 3 as described in the previous section. Figure 3 shows the excellent quality of the fit at four selected wavelengths. The estimated kinetic parameters appear in Table 1, and the spectra in Figure 4 represent the molar absorptivities of the four electronic species at the 13 wavelengths. Figure 5 shows the temporal evolution of the concentration of different electronic species in mechanism 3 calculated with the parameters obtained from the global fit in the 400-1350 nm wavelength range.

To quantitatively test the goodness of fit, we used the simple statistics

$$
Q=100\left(1-\frac{S_{\text {residual }}^{2}}{S_{y}^{2}}\right)
$$

which gives the "quality of the fit" in percentage. In the formula, $S_{\text {residual }}^{2}$ is the sum of squared residuals (i.e., the 


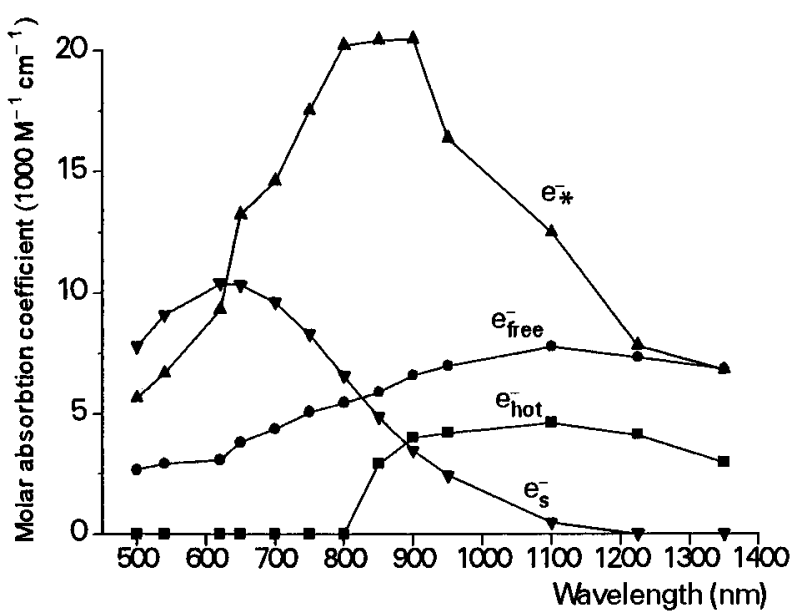

Figure 4. Spectra of the four species included in mechanism 3 for electron solvation in neat methanol at room temperature. Spectral curves are labeled with the corresponding species. The spectrum of $\mathrm{e}_{\mathrm{s}}^{-}$is taken from the literature, ${ }^{31}$ while the other three are estimated values from the present analysis. The spectrum labeled $\mathrm{e}_{\mathrm{hot}}^{-}$is assigned to all thermalizing species from $\mathrm{e}_{1}^{-}$to $\mathrm{e}_{n}^{-}$in mechanism 3 .

differences between the observed and the fitted values) and $S_{y}^{2}$ is the overall variance of the observed values. This gives the percentage of overall variation explained by the fitted model or, in other words, the explanatory power of the model. The percentage $Q$ is useful to compare the quality of fit of different models to the same data set. We compared the $Q$ values obtained with mechanism 3 to those of a "best fit" (a suitably flexible function with no strict physical sense) and found that the $Q$ values of $98-99 \%$ for mechanism 3 are typically smaller by only less than half percent than the $Q$ 's of the "best fit". The 1225 and $1350 \mathrm{~nm}$ traces were somewhat different: they resulted in only 93 and $94.5 \% Q$ values for both mechanism 3 and the "best fit". This is due to the greater experimental error in these kinetic traces resulting in considerably higher $S_{\text {residual }}^{2}$ in the numerator of the fraction of expression 4. Such high $Q$ values indicate that the random error is only of the magnitude of the experimental uncertainties.

We also tested if the distribution of the errors were strictly random at individual wavelengths. To this end, we performed Durbin-Watson D-tests ${ }^{32}$ on subsequent residuals, which revealed a statistically significant serial positive correlation in 8 of the 13 cases. This is probably a consequence of the applied numerical approach. Global fitting procedure may result in excellent fits for a few wavelengths causing slightly less satisfying fits for others. However, femtosecond laser experiments might produce slow periodic fluctuations in the measured kinetic traces and it is not yet clear how this could contribute to serial correlations indicated by the D-test. The global fit of mechanism 3 to the experimental kinetic traces at the 13 wavelengths can be considered excellent, so we can state that mechanism 3 is fully consistent with the experimental data.

Besides the calculation of characteristic times (thermalization, localization, solvation) and branching ratio, which we will discuss afterward, one of the benefits provided by fitting model 3 to the data is that for the first time we were able to extract the absorption spectra of all electronic species taking part in the solvation process. Previous models excluded any absorption from nonlocalized electrons in this wavelength domain (nearIR and visible). Some interesting spectral properties can be observed on Figure 3. As expected, the two nonlocalized species have maxima at lower energy than $\mathrm{e}_{*}^{-}$and $\mathrm{e}_{\mathrm{s}}^{-}$. The molar absorption coefficients are far from negligible even in the visible. In fact even as low as $500 \mathrm{~nm}$ the sum of the values
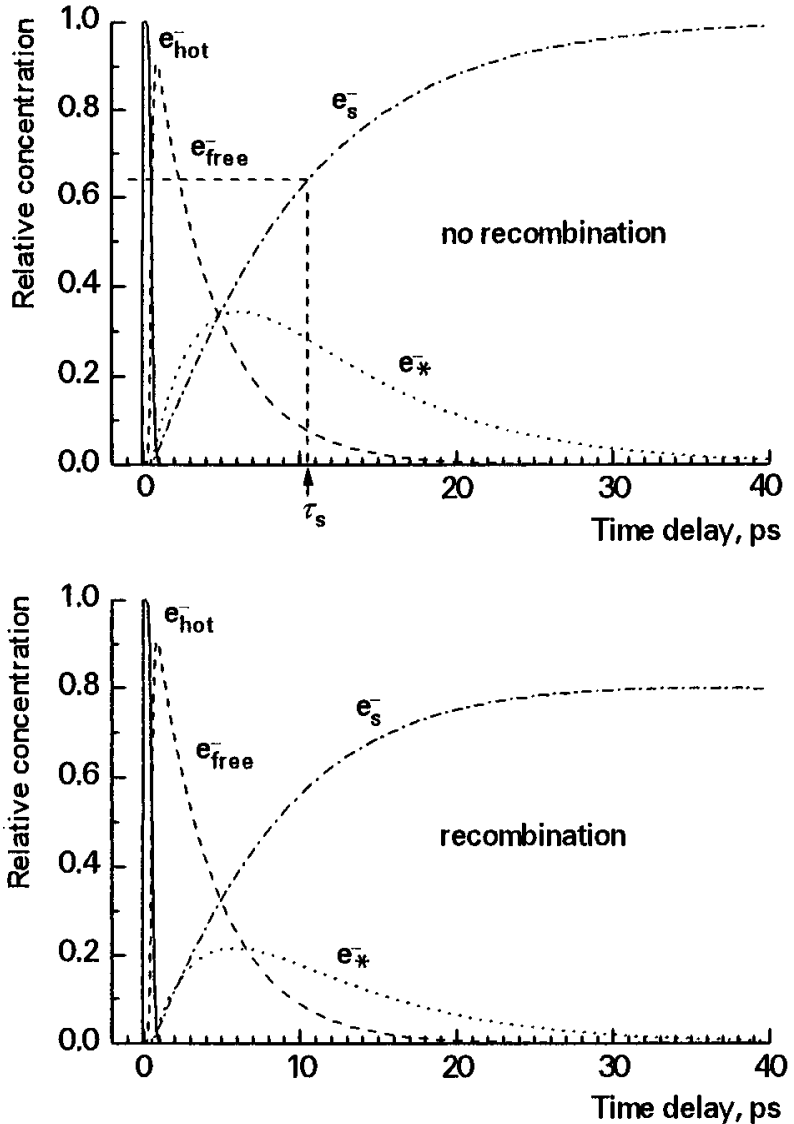

Figure 5. Temporal evolution of the relative concentration of the electronic species according to mechanism 3 for electron solvation in neat methanol at $294 \mathrm{~K}$, including geminate recombination. Note the fast decay of the $e_{\text {hot }}^{-}$and the relatively long persistence of the $e_{\text {free }}^{-}$ species. The lower diagram shows the evolution during the experiment, including the effect of recombination reducing the electron concentration by $\sim 20 \%$ after $40 \mathrm{ps}$. The upper diagram shows this evolution without the effect of recombination, thus enabling the determination of a correct overall solvation time $\tau_{\mathrm{s}}$, the time necessary to solvate the $(1-1 / \mathrm{e})$ fraction of the initially generated electrons.

for the free electron and the $\mathrm{e}_{*}^{-}$excited state exceeds that of the fully solvated electron.

In order to compare our present results to previous findings on electron solvation in the literature, we should produce parameters of the same nature as has been published before. The usual characteristic time was single exponential decay time at some wavelengths, or rise time at other wavelengths, ${ }^{2-9,18,19}$ and a solvation time, which has been deduced from the rise and decay times, according to some model assumptions. As both the experimental data and the applied statistical method allowed for a much more sophisticated way of data analysis, we directly calculate the parameters of the complex model mechanism 3. Nevertheless, it is easy to get a single solvation time from our data. The characteristic time of solvation, $\tau_{\mathrm{s}}$, means that only $1 / \mathrm{e}$ portion of the initially generated electrons is "unsolvated", i.e., $1-1 / \mathrm{e}$ portion is already solvated. The corresponding time can readily be obtained, as illustrated in Figure 5a. The resulting $\tau_{\mathrm{s}}=10.3 \mathrm{ps}$ compares very well with recent values centered around $10-10.7$ ps. ${ }^{19 b}$ However, we can also analyze our deconvoluted kinetic curves at individual wavelengths, after the removal of the effect of recombination, and get single experimental "rise time" and "decay time" at different wavelengths. In doing so, it should be emphasized that there is a coupling (or, in statistical terms, a correlation) between the kinetic parameters and the absorptivities of different species, contributing to the rise or decay time in a complicated 
way. Nevertheless, decay or rise can excellently be fitted by a simple exponential practically for all kinetic traces in a considerably wide temporal range, and the emerging characteristic times are not that much different. In the case of the kinetic curves discussed in this paper, the decay time varies from 9.5 to 10.6 ps for the wavelengths between 700 and 1350 $\mathrm{nm}$, while the rise time varies between 4.5 and 2.5 ps between 500 and $650 \mathrm{~nm}$. Obviously, decay times are closely related to the overall solvation time $\tau_{\mathrm{s}}$, while it is not so evident to assign the rise time to one single dynamic event, not even to the overall localization time. Accordingly, many of the early studies $^{2-5}$ considered the decay time at higher wavelengths as $\tau_{\mathrm{s}}$, and those who rather used the rise time at lower wavelengths to assign as $\tau_{\mathrm{s}}$ have found that it changes considerably from one wavelength to the other. ${ }^{7,8}$

Ever since the first radiolysis studies of electron solvation in polar liquids, a striking resemblance of the solvation time with dielectric relaxation times was noticed and discussed. Extensive discussion of this resemblance can be found in refs 5, 18-19, and 33. It is the so-called "constant charge" dielectric relaxation whose characteristic time is close to solvation times found in alcohols. In the case of methanol, both $\tau_{\mathrm{D}_{2}}$, the relaxation time related to the rotation of the non-H-bonded monomer, and $\tau_{\mathrm{L}_{1}}$, the longitudinal relaxation time related to the breaking of the H-bonds are typically between 9 and 12 ps, quite close to the overall solvation time. We think that dielectric relaxation parameters reflect more of a "continuum" behavior of the medium hence the correspondence with the "overall" solvation time, which reflects the whole of the complex solvation procedure. On the other hand, an electron probes more a molecular and microscopic environment, so the characteristic times of elementary dynamic events might be quite different from the essentially continuum-based dielectric relaxation times. Or, as Chase and Hunt ${ }^{5}$ put it: "It is possible that this correspondence is just a coincidence and that the theory of the constant charge modification to $\tau_{1}$ is correct in principle but not in its detailed application to the dynamics of solvation", and "There is a fundamental difference in the two processes; in dielectric relaxation the reorienting field is external to the medium while in electron solvation it is internal."

Electron solvation in $\mathrm{H}_{2} \mathrm{O}$ became the first testing ground for model $3 .{ }^{28}$ The experimental data of Migus et al..$^{10}$ has been used for that purpose. Table 1 lists the calculated parameters for both molecules. According to the data shown there, localization $\left(T_{\mathrm{loc}}\right)$ is 50 times slower in methanol than in water. Characteristic times of the two-step channels $\left(T_{1}\right.$ and $\left.T_{2}\right)$ also reflect this difference: they are 59 and 26 times longer for methanol, respectively. These important differences might originate in differences between the structural and dynamic properties of the hydrogen-bonded network in methanol and water. The large scale three-dimensional H-bond network in water is much quicker to respond to the sudden appearance of an electron than the unidimensional H-bond network in methanol. In addition, methanol molecules are twice heavier than water molecules. This could perhaps explain part of the roughly 50-fold difference in localization and solvation rates. In a recent paper, Hilczer and Tachiya reported a simulation study of preexisting electron trap dynamics in pure methanol. ${ }^{34}$ According to their results, only less than $20 \%$ of the pre-existing traps survives for more than $100 \mathrm{fs,} \mathrm{which} \mathrm{would} \mathrm{"justify} \mathrm{the}$ commonly assumed "trap-seeking" mechanism of electron trapping". Now, if this "trap-seeking" dynamic is radically slower in methanol than in water, it could explain such a great difference in localization times. We think that the essentially three-dimensional H-bond network in water could be coupled to the quasi-free electron states in a manner which would facilitate concerted ultrafast rearrangements of existing traps as a response to the presence of the electron in the time scale of the $\mathrm{OH}$-vibrations, an order of magnitude of $10 \mathrm{fs}$. In methanol, the H-bond network is essentially one-dimensional, so the reorientational response in methanol, which should include at least librational motions of the $\mathrm{CH}_{3} \mathrm{OH}$ molecules, should be much slower than the $10 \mathrm{fs} \mathrm{OH}$-vibration period.

Another major discrepancy between these two polar solvents is that thermalization $\left(n T_{\text {th }}\right)$ is 7 times slower in methanol than in water. Thermalization might not depend so much on the $\mathrm{H}$-bonded structure. If we make the assumption that the energy of the electron is mainly deposited in the $\mathrm{OH}$ vibrational modes, a major part of the 7-fold difference in thermalization rates is already accounted for by comparing $\mathrm{OH}$-bond densities in the two liquids. At room temperature, there are $111 \mathrm{~mol}$ of $\mathrm{OH} /$ $\mathrm{dm}^{3}$ in water and $24.6 \mathrm{~mol}$ of $\mathrm{OH} / \mathrm{dm}^{3}$ in methanol, i.e., 4.5 times more $\mathrm{OH}$ bonds in water than in methanol. (This argument would not hold if electron energy deposition in the $\mathrm{CH}$ vibrations is also effective.)

At this point, the question could be raised: are free electrons really formed during the ionization of pure methanol with 2-eV laser pulses? Recent papers published on the study of multiphoton ionization in water found that this is not the case in many instances. ${ }^{35,36}$ However, since the existence of free electrons was also supposed in the original paper reporting these experiments ${ }^{1}$ and mechanism 4 contains this supposition, we kept this part of the mechanism. It should be mentioned that more recent measurements in the same laboratory on electron solvation in pure water made it highly probable that five $2-\mathrm{eV}$ photons were needed to produce the observed electrons. ${ }^{37}$ As the same pulses were used for the experiments analyzed in this paper, we could suppose that $10 \mathrm{eV}$ should have been enough to ionize methanol to give free electrons.

There is also a similarity between the two solvents: branching ratios are only slightly different. The two-step channel to direct solvation path proportion for methanol is $67: 33$, while it is 78 : 22 for water, so direct solvation is increased to one-third compared to one-fourth in water.

Let us examine mechanisms 3 and 4 that both numerically reproduce the experimental data fully and excellently. Both favor two different solvation paths one of which is a direct localization in the final electronic state while the other goes through an intermediate state. The passage from the intermediate state to the final state is roughly the same in both cases: 8.4 vs $6.1 \mathrm{ps}$, respectively. On all other aspects the two models largely disagree. The discrepancies stem from the fact that in one instance absorption from the nonlocalized electrons in the spectral region covered $(400-1350 \mathrm{~nm})$ is insignificant. On the contrary, model 3 has to consider major molar absorption coefficients from these species in order to explain important signal attenuation from quite low visible wavelengths to the near-IR at early times. However, it may be argued that this spectral feature has also been observed in glassy ethanol at 4 $\mathrm{K}$, where there is a big overlap of the spectra of the presolvated electron with the fully solvated one in the visible wavelength range and the IR-band of the presolvated species has a large visible tail stretching well below $400 \mathrm{~nm} .{ }^{9,38}$

Localization times are also very different. After 1 ps almost every electron is localized from the model 4 point of view but only a few percent in model 3 (see Figure $5 \mathrm{~b}$ ) where $T_{\text {loc }}=3.9$ ps. The hybrid model allows for relaxation of both electronic species which is reflected in a continuous shift of their spectra $\left(T_{\text {cont }}=13.6 \mathrm{ps}\right)$. For the other model all 4 spectra are spectrally insensitive to solvent relaxation. Finally, mechanism 4 can be 
essentially decomposed in two stages: a fast localization (mainly via the direct path $<1 \mathrm{ps}$ ) and then a slow stepwise transition $\left(T_{\text {step }}=6.1 \mathrm{ps}\right)$. That is not the case for mechanism 3 where both channels of solvation are slow processes, the direct solvation having a characteristic time $T_{3}=11.7 \mathrm{ps}$ and the indirect route being a sequence of $T_{1}=5.9 \mathrm{ps}$ and $T_{2}=8.4 \mathrm{ps}$.

There is an interesting feature of mechanism 3 , namely, that it accounts in detail for the "history" of the electron prior to localization, while the previously published model (4) only states that "within $1 \mathrm{ps}$, the quasi-free electrons $\left(\mathrm{e}_{\mathrm{qf}}^{-}\right)$become distributed in two localized electron-solvent configuration states." Therefore, mechanism 3 can be compared with another proposal for electron solvation put forward by Lewis and Jonah ${ }^{39}$ when discussing their results of electron scavenging in alcohols. They proposed the mechanism

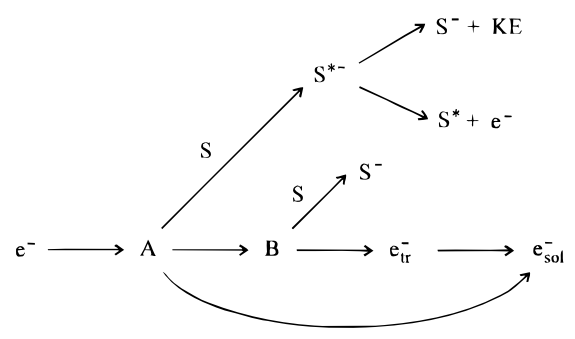

where the "horizontal" part from $\mathrm{e}^{-}$to $\mathrm{e}_{\text {sol }}^{-}$is the solvation mechanism, and state A has a higher (kinetic) energy than state $B$, both being (nonlocalized) precursors of $\mathrm{e}_{\mathrm{tr}}^{-}$and/or $\mathrm{e}_{\mathrm{sol}}^{-}$. From the scavenging results ("vertical" part of the above mechanism) they concluded that there is a "fast solvation channel", $A$ to $\mathrm{e}_{\text {sol }}^{-}$, and a "slow solvation channel", $\mathrm{B}$ to $\mathrm{e}_{\text {sol }}^{-}$, where A and B are both "dry" (i.e., nonlocalized) electrons. Mechanism 3 is not that much different from mechanism 6, as we have to put only the branching from $\mathrm{e}_{\text {free }}^{-}$back to $\mathrm{e}_{n}^{-}$, $\mathrm{e}_{n-1}^{-}$, or even further in mechanism 3 to get an equivalent mechanism to (6). Work is in progress to change (3) accordingly and analyze present data to get kinetic parameters corresponding to mechanism 6 .

Since both models 3 and 4 numerically reproduce the subpicosecond pump-and-probe kinetic data, input from other independent experimental sources will prove valuable. The debate would greatly benefit from fresh and more accurate results on the localization time in methanol. This information could decide the issue. Scavenging experiments could be extremely useful not only to study branching details of the solvation mechanisms but also to decide whether the continuous blue shift is an essential part of the electron solvation in methanol. Some new carefully designed scavenging experiments may explore the details of spectral changes during electron solvation, together with a detailed analysis of the possibility of branching during thermalization prior to electron localization. Other experiments like photoelectron injection in polar liquids where thermalizing distances can be evaluated may help in giving new insight on the solvation process.

\section{Conclusion}

We have confirmed on the basis of pump-and-probe laser measurements of electron solvation in neat methanol that a simple two-step mechanism cannot interpret the kinetic curves in the entire wavelength range from 400 to $1350 \mathrm{~nm}$, though it is applicable in a smaller wavelength range. We suppose that the successful interpretation of the same kind of kinetic results on the basis of the two-step mechanism by Shi et al. is due either to the narrow wavelength range $(625-900 \mathrm{~nm})$ studied or to a different ionization mechanism ${ }^{35,36,40}$ in their experiments than in the present study.

It has been found that, in addition to the solvation mechanism published in a previous paper $^{1}$ which included continuously blue-shifting spectra of the two localized species, another mechanism without continuous spectral blue shift involving significant absorption of the presolvated electron species in the visible and the near-IR can also numerically describe the measured kinetic traces in the entire 400-1350 nm wavelength range. This mechanism, the equivalent of what has been found in diabatic quantum-dynamic simulations, ${ }^{24,27}$ comprises a thermalization prior to a branching localization, leading directly or via a presolvated species to the fully solvated electron (see scheme 3 in the Introduction). A comparison of electron solvation parameters of the same mechanism in water ${ }^{28}$ to the present results shows that thermalization is 7 times slower in methanol, while localization and final solvation are respectively 50 and 22 times slower than in water. The two models exhibit almost incompatible conceptual views which are reflected in sizable differences in localization time. To shed new light on the matter, we anticipate some new scavenging experiments or other experimental results which can more accurately determine thermalization and localization times in methanol.

Acknowledgment. The authors express their special thanks for C. Pépin, D. Houde, and T. Goulet from Sherbrooke University for providing detailed kinetic data, and for heated and fruitful discussions. The support for cooperation of NATO by the Linkage Grant No. LG921374 is also acknowledged. L. Turi is a Magyary Zoltán Fellow (Foundation for Hungarian Education, Ministry of Education, Hungary), which is gratefully acknowledged. E. Keszei and L. Turi are recipients of grant supports from the National Research Fund of Hungary under Contract Nos. OTKA T019396 and OTKA F019474.

\section{References and Notes}

(1) Pépin, C.; Goulet, T.; Houde D.; Jay-Gerin, J.-P. J. Phys. Chem. 1994, 98, 7009 .

(2) Baxendale, J. H.; Wardman, P. Nature 1971, 230, 450.

(3) Baxendale, J. H.; Wardman, P. J. Chem. Soc., Faraday Trans. 1 1973, 69, 584. 8,621

(4) Baxendale, J. H.; Sharpe, P. H. G. Int. J. Radiat. Phys. Chem. 1976,

(5) Chase, W. J.; Hunt, J. W. J. Phys. Chem. 1975, 79, 2835.

(6) Klassen, N. V.; Gillis, H. A.; Teather, G. G.; Kevan, L. J. Chem. Phys. 1975, 62, 2474.

(7) Gilles, L.; Bono, M. R.; Schmidt, M. Can. J. Chem. 1977, 55, 2003

(8) Okazaki, K.; Freeman, G. R. Can. J. Chem. 1978, 56, 2305.

(9) Ogasawara, M.; Shimizu, K.; Yoshida, H. Radiat. Phys. Chem. 1981, $17,331$.

(10) Migus, A.; Gauduel, Y.; Martin, J. L.; Antonetti, A. Phys. Rev. Lett. 1987, 58, 1559.

(11) Gauduel, Y.; Pommeret, S.; Migus, A.; Antonetti, A. Radiat. Phys. Chem. 1989, 34, 5; J. Phys. Chem. 1989, 93, 5.

(12) Gauduel, Y.; Pommeret, S.; Migus, A.; Antonetti, A. J. Am. Chem. Soc. 1990, 112, 2925.

(13) Long, F. H.; Lu, H.; Eisenthal, K. B. J. Chem. Phys. 1989, 91, 4413.

(14) Long, F. H.; Lu, H.; Eisenthal, K. B. Phys. Rev. Lett. 1990, 64, 1469.

(15) Long, F. H.; Lu, H.; Eisenthal, K. B. J. Opt. Soc. Am. B 1990, 7, 1511.

(16) Pommeret, S.; Antonetti, A.; Gauduel, Y. J. Am. Chem. Soc. 1991, $113,9105$.

(17) Gauduel, Y.; Pommeret, S.; Antonetti, A. J. Phys. Chem. 1993, 97,134

(18) Hirata, Y.; Murata, N.; Tanioka, Y.; Mataga, N. J. Phys. Chem. 1989, 93, 4527. Hirata, Y.; Mataga, N. J. Phys. Chem. 1990, 94, 8503.

(19) Hirata, Y.; Mataga, N. J. Phys. Chem. 1991, 95, 9067. Hirata, Y.; Mataga, N. Prog. React. Kinet. 1993, 18, 273.

(20) Walhout, P. K.; Alfano, J. C.; Kimura, Y.; Silva, C.; Reid, P. J.; Barbara, P. F. Chem. Phys. Lett. 1995, 232, 135. 4413. 
(22) Neria, E.; Nitzan, A.; Barnett, R. N.; Landman, U. Phys. Rev. Lett. 1991, 67, 1011 .

(23) Webster, F. J.; Schnitker, J.; Friedrichs, M. S.; Friesner, R. A.; Rossky, P. J. Phys. Rev. Lett. 1991, 66, 3172.

(24) Murphrey, T. H.; Rossky, P. J. J. Chem. Phys. 1993, 99, 515.

(25) Schwartz, B. J.; Rossky, P. J. Phys. Rev. Lett. 1994, 72, 3282.

(26) Schwartz, B. J.; Rossky, P. J. J. Phys. Chem. 1994, 98, 4489.

(27) Keszei, E.; Nagy, S.; Murphrey, T. H.; Rossky, P. J. J. Chem. Phys. 1993, 99, 2004

(28) Keszei, E.; Murphrey, T. H.; Rossky, P. J. J. Phys. Chem. 1995, 99,22 .

(29) Shi, X.; Long, F. H.; Lu, H.; Eisenthal, K. B. J. Phys. Chem. 1995, 99, 6917.

(30) DeNoyer, L. K.; Dodd, J. G. Am. Lab. 1991, 23, 24D

(31) Jou, F.-Y.; Freeman, G. R. J. Phys. Chem. 1977, 81, 909. Jou, F.Y.; Freeman, G. R. Can. J. Chem. 1979, 57, 591.
(32) Durbin, J.; Watson, G. S. Biometrika 1950, 37, 409; 1951, 38, 159. Himmelblau, D. M. In Process analysis by statistical methods; Wiley: New York, 1970.

(33) Kenney-Wallace, G. A.; Jonah, C. D. J. Phys. Chem. 1982, 86, 2572.

(34) Hilczer, M.; Tachiya, M. J. Phys. Chem. 1996, 100, 7691.

(35) Crowell, R. A.; Bartels, D. M. J. Phys. Chem. 1996, 100, 17940.

(36) Reuther, A.; Lauberau, A.; Nikogosyan, D. N. J. Phys. Chem. 1996, $100,16794$.

(37) Pépin, C.; Goulet, T.; Houde D.; Jay-Gerin, J.-P. J. Phys. Chem., in press.

(38) Hase, H.; Warashina, T.; Noda, M.; Namiki, A.;Higashimura, T. J. Chem. Phys. 1972, 57, 1039.

(39) Lewis, M. A.; Jonah, C. D. J. Phys. Chem. 1986, 90, 5367.

(40) Sander, M.; Brummund, U.; Luther, K.; Troe, J. Ber. Bunsenges. Phys. Chem. 1992, 96, 1486. 